\title{
The 2dF Galaxy Redshift Survey: the bias of galaxies and the density of the Universe
}

\author{
Licia Verde, ${ }^{1,2 \star}$ Alan F. Heavens, ${ }^{3}$ Will J. Percival, ${ }^{3}$ Sabino Matarrese, ${ }^{4}$ \\ Carlton M. Baugh, ${ }^{5}$ Joss Bland-Hawthorn, ${ }^{6}$ Terry Bridges, ${ }^{6}$ Russell Cannon, ${ }^{6}$ \\ Shaun Cole,${ }^{5}$ Matthew Colless, ${ }^{7}$ Chris Collins, ${ }^{8}$ Warrick Couch, ${ }^{9}$ Gavin Dalton, ${ }^{10}$ \\ Roberto De Propris, ${ }^{9}$ Simon P. Driver, ${ }^{11}$ George Efstathiou, ${ }^{12}$ Richard S. Ellis, ${ }^{13}$ \\ Carlos S. Frenk, ${ }^{5}$ Karl Glazebrook, ${ }^{14}$ Carole Jackson, ${ }^{7}$ Ofer Lahav, ${ }^{12}$ Ian Lewis, ${ }^{10}$ \\ Stuart Lumsden, ${ }^{15}$ Steve Maddox, ${ }^{16}$ Darren Madgwick, ${ }^{12}$ Peder Norberg, ${ }^{5}$ \\ John A. Peacock, ${ }^{3}$ Bruce A. Peterson, ${ }^{7}$ Will Sutherland ${ }^{3}$ and Keith Taylor ${ }^{6}$ \\ ${ }^{1}$ Department of Physics and Astronomy, Rutgers University, 136 Frelinghuysen Road, Piscataway, NJ 08854-8019, USA \\ ${ }^{2}$ Princeton University Observatory, Princeton, NJ 08544, USA \\ ${ }^{3}$ Institute for Astronomy, University of Edinburgh, Royal Observatory, Blackford Hill, Edinburgh EH9 $3 H \mathrm{HJ}$ \\ ${ }^{4}$ Dipartimento di Fisica, 'G. Galilei', Università di Padova, and INFN, Sezione di Padova, via Marzolo 8, I-35131 Padova, Italy \\ ${ }^{5}$ Department of Physics, University of Durham, South Road, Durham DH1 $3 \mathrm{LE}$ \\ ${ }^{6}$ Anglo-Australian Observatory, PO Box 296, Epping, NSW 2121, Australia \\ ${ }^{7}$ Research School of Astronomy \& Astrophysics, The Australian National University, Weston Creek, ACT 2611, Australia \\ ${ }^{8}$ Astrophysics Research Institute, Liverpool John Moores University, 12 Quays House, Birkenhead L14 ILD \\ ${ }^{9}$ Department of Astrophysics, University of New South Wales, Sydney, NSW 2052, Australia \\ ${ }^{10}$ Department of Physics, University of Oxford, Keble Road, Oxford OX1 3RH \\ ${ }^{11}$ School of Physics and Astronomy, University of St Andrews, North Haugh, St Andrews, Fife KY6 9SS \\ ${ }^{12}$ Institute of Astronomy, University of Cambridge, Madingley Road, Cambridge CB3 OHA \\ ${ }^{13}$ Department of Astronomy, California Institute of Technology, Pasadena, CA 91125, USA \\ ${ }^{14}$ Department of Physics \& Astronomy, Johns Hopkins University, Baltimore, MD 21218-2686, USA \\ ${ }^{15}$ Department of Physics, University of Leeds, Woodhouse Lane, Leeds LS2 9JT \\ ${ }^{16}$ School of Physics and Astronomy, University of Nottingham, Nottingham NG7 2RD
}

Accepted 2002 April 26. Received 2002 March 15; in original form 2001 December 11

\begin{abstract}
We compute the bispectrum of the 2dF Galaxy Redshift Survey (2dFGRS) and use it to measure the bias parameter of the galaxies. This parameter quantifies the strength of clustering of the galaxies relative to the mass in the Universe. By analysing $80 \times 10^{6}$ triangle configurations in the wavenumber range $0.1<k<0.5 \mathrm{~h} \mathrm{Mpc}^{-1}$ (i.e. on scales roughly between 5 and $30 \mathrm{~h}^{-1}$ $\mathrm{Mpc}$ ) we find that the linear bias parameter is consistent with unity: $b_{1}=1.04 \pm 0.11$, and the quadratic (non-linear) bias is consistent with zero: $b_{2}=-0.054 \pm 0.08$. Thus, at least on large scales, optically selected galaxies do indeed trace the underlying mass distribution. The bias parameter can be combined with the $2 \mathrm{dFGRS}$ measurement of the redshift distortion parameter $\beta \simeq \Omega_{\mathrm{m}}^{0.6} / b_{1}$, to yield $\Omega_{\mathrm{m}}=0.27 \pm 0.06$ for the matter density of the Universe, a result that is determined entirely from this survey, independent of other data sets. Our measurement of the matter density of the Universe should be interpreted as $\Omega_{\mathrm{m}}$ at the effective redshift of the survey $(z=0.17)$.
\end{abstract}

Key words: galaxies: clusters: general - cosmological parameters - large-scale structure of Universe.

\section{INTRODUCTION}

Clustering of mass in the Universe is believed to be a result of amplification by gravitational instability of small perturbations generated

^E-mail: 1verde@astro.princeton.edu in the early Universe. Comparison with theoretical predictions offers the chance to test models of generation of the perturbations, as well as putting important constraints on cosmological parameters, which control the growth rate of the perturbations. A fundamental limitation on such a comparison has been that theoretical models predict the clustering properties of the mass in the Universe, and yet 
we have few direct measures of mass observationally. More readily observable is the distribution of luminous objects such as galaxies, so to compare with theory one has to determine, or assume, the relationship between the clustering of mass and the clustering of galaxies. In general, one will expect these to differ, because the efficiency of galaxy formation may depend in some non-trivial way on the underlying mass distribution. The idea that structures may be 'biased' tracers of the mass distribution goes back to Kaiser (1984), who explained the high clustering strength of Abell clusters as being a result of their forming in high-density regions of the Universe. In addition, observations indicating that different types of galaxy cluster differently (e.g. Dressler 1980; Postman \& Geller 1984; White, Tully \& Davis 1988; Hamilton 1988; Lahav, Nemiroff \& Piran 1990; Lahav \& Saslaw 1992) show that they cannot all be unbiased tracers of the mass. Bias became an attractive way to reconcile the low velocities of galaxies with the high-density Einstein-de Sitter model favoured in the 1980s (e.g Davis et al. 1985), but after the Cosmic Background Explorer (COBE) determined the amplitude of primordial fluctuations on large scales Smoot et al. (1992), the 'standard' biased cold dark matter (CDM) model became less popular. With the advent of more detailed data sets for the cosmic microwave background (CMB) and large-scale structure, it is possible to investigate and constrain a wider range of galaxy formation models, and an unknown bias relation adds uncertainty to the process.

Since the efficiency of galaxy formation is not well understood theoretically, it makes sense to try to measure it empirically from observations. When the perturbations are small (or on large, linear scales), it is difficult to do this: there is a degeneracy between the unknown amplitude of the matter power spectrum $P(k)$ and the degree of bias, $b$, defined such that the galaxy power spectrum is $P_{\mathrm{g}}(k) \equiv b^{2} P(k)$. In principle, $b$ may be a function of scale, through the wavenumber $k$. At later times (or on smaller scales), however, the degeneracy is lifted by non-linear effects. One feature of non-linear gravitational evolution is that the overdensity field $\delta(\boldsymbol{x}) \equiv[\rho(\boldsymbol{x})-\bar{\rho}] / \bar{\rho}$ becomes progressively more skewed towards high density. In principle skewness could also arise from nonGaussian initial conditions; in practice this can be neglected (Verde et al. 2000), since CMB fluctuations are consistent with Gaussian initial conditions (Komatsu et al. 2002; Santos et al. 2001). One can thus hope to exploit the gravitational skewness, but skewness could equally well arise from biasing, e.g. from a galaxy formation efficiency that increased at dense points in the mass field. It is nevertheless possible to distinguish these two effects by considering the shapes of isodensity regions. If the field is unbiased, then the shapes of isodensity contours become flattened, as gravitational instability accelerates collapse along the short axis of structures, leading to sheet-like and filamentary structures (e.g. Zeldovich 1970). If the galaxy field is highly biased with the same power spectrum, however, the underlying mass field is of low amplitude, and thus will be expected to be close to the initial field, which is assumed to be Gaussian. These fields do not have highly flattened isodensity contours, as bias does not flatten the contours; for example, Eulerian bias preserves the contour shape. Thus there is a difference, which could be detected, for example, by studying the three-point correlation function. In this paper, we exploit this effect in Fourier space rather than in real space, by analysing the bispectrum: $\left\langle\delta_{k_{1}} \delta_{k_{2}} \delta_{k_{3}}\right\rangle$, where $\delta_{\boldsymbol{k}}$ is the Fourier transform of the galaxy overdensity field. The theory for the bispectrum is set out in Fry (1994), Hivon et al. (1995), Matarrese, Verde \& Heavens (1997), Verde et al. (1998), Scoccimarro et al. (1998), Scoccimarro, Couchman \& Frieman (1999) and Scoccimarro (2000).
The galaxy survey we use is the Anglo-Australian Telescope $2^{\circ}$ Field Galaxy Redshift Survey (Colless et al. 2001), as compiled in 2001 February. It was created with the $2 \mathrm{dF}$ multifibre spectrograph on the Anglo-Australian Telescope (Lewis et al. 2002), and currently consists of over 200000 galaxies with redshifts up to about $z=0.3$, broadly in two regions centred near the south and north galactic poles. See http://www.mso.anu.edu.au/2dFGRS/ for further details. It is the first survey that is large enough to put tight constraints on the bias parameter, as previous surveys are too shallow or too sparse. In this paper, we use 127000 galaxies from the 2001 February compilation of the catalogue, truncated at $0.03<z<0.25$.

The outline of the paper is as follows. In Section 2 we review the theory of growth of the bispectrum through gravitational instability, and discuss briefly the effects of redshift-space distortions; in Section 3 we illustrate our method of measuring the bias parameter. This method uses a new estimator of the bias parameter, which allows us to analyse many millions of $\boldsymbol{k}$-vector triplets, thus drastically improving the signal-to-noise ratio. In Section 4 we test the performance of the method. Finally, in Section 5 we present our results and in Section 6 we discuss the implications of these results. An Appendix presents and describes in detail our new estimator of the bias parameter.

\section{THE BISPECTRUM IN REAL AND REDSHIFT SPACE}

The statistic we use to measure the bias of the galaxies is the bispectrum $B$, which is related to the three-point correlation function in Fourier space. For the mass, this is defined by

$\left\langle\delta_{\boldsymbol{k}_{1}} \delta_{\boldsymbol{k}_{2}} \delta_{\boldsymbol{k}_{3}}\right\rangle \equiv(2 \pi)^{3} B\left(\boldsymbol{k}_{1}, \boldsymbol{k}_{2}, \boldsymbol{k}_{3}\right) \delta^{\mathrm{D}}\left(\boldsymbol{k}_{1}+\boldsymbol{k}_{2}+\boldsymbol{k}_{3}\right)$,

where $\delta_{\boldsymbol{k}} \equiv \int \mathrm{d}^{3} \boldsymbol{x} \delta(\boldsymbol{x}) \exp (-\mathrm{i} \boldsymbol{k} \cdot \boldsymbol{x})$ is the Fourier transform of the mass overdensity $\delta(\boldsymbol{x}) \equiv \rho(\boldsymbol{x}) / \bar{\rho}-1$ and $\delta^{\mathrm{D}}$ is the Dirac delta function, which shows that the bispectrum can be non-zero only if the $\boldsymbol{k}$-vectors close to form a triangle.

The power spectrum $P$ is similarly defined by

$\left\langle\delta_{\boldsymbol{k}} \delta_{\boldsymbol{k}^{\prime}}\right\rangle \equiv(2 \pi)^{3} P(k) \delta^{\mathrm{D}}\left(\boldsymbol{k}+\boldsymbol{k}^{\prime}\right)$.

Analogous relations hold for the power spectrum and bispectrum of the galaxy distribution. We assume that the mass overdensity is a Gaussian random field initially, as closely predicted by inflationary early-universe models. Thus, at asymptotically early times the bispectrum is zero by symmetry. As gravitational instability develops, the field becomes asymmetric, because non-linear effects skew the density field to high densities. In this way, a non-zero bispectrum develops. In the mildly non-linear regime, we use second-order perturbation theory to compute the expected bispectrum. To second order (in the overdensity $\delta$ ) the Fourier coefficients develop a nonlinear component which is proportional to $\delta^{2}$, so the leading-order term in the bispectrum grows as $\delta^{4}$. Since the $2 \mathrm{dFGRS}$ is not a survey of mass density, to interpret the bispectrum measured from the survey we must make some assumption concerning the distribution of mass relative to the distribution of galaxies. To date, this uncertainty in the relationship between the mass and the galaxy distribution has placed a limitation on the usefulness of galaxy catalogues as a probe of cosmology. We make the assumption that the galaxy overdensity field $\delta_{\mathrm{g}}$ is related to the underlying mass overdensity by some deterministic function, which we expand in a Taylor series as (cf. Fry \& Gaztanaga 1993)

$\delta_{\mathrm{g}}=\sum_{i=0}^{\infty} \frac{b_{i} \delta^{i}}{i !}$. 
We must keep terms up to $i=2$, since these enter in the bispectrum at the same level as second-order perturbation theory growth terms, and we ignore higher-order terms. In order for $\delta_{\mathrm{g}}$ to have zero mean, there is a (calculable) $b_{0}$ term, but we ignore it as it only contributes to $\boldsymbol{k}=\mathbf{0} ; b_{1}$ is the linear bias parameter and $b_{2}$ is the quadratic bias parameter. $A$ non-zero $b_{2}$ would indicate non-linear biasing of galaxies with respect to mass. Both of these parameters are estimated in this paper from the 2dFGRS.

In real space, the two effects of non-linear growth and non-linear bias contribute terms to a non-zero bispectrum:

$B\left(\boldsymbol{k}_{1}, \boldsymbol{k}_{2}, \boldsymbol{k}_{3}\right)=P_{\mathrm{g}}\left(k_{1}\right) P_{\mathrm{g}}\left(k_{2}\right)\left[\frac{J\left(\boldsymbol{k}_{1}, \boldsymbol{k}_{2}\right)}{b_{1}}+\frac{b_{2}}{b_{1}^{2}}\right]+$ cyc.,

where there are two additional cyclic terms $(2,3)$ and $(3,1)$. Details of the theory leading to (4) may be found in, for example, Matarrese et al. (1997). We assume here that the galaxy power spectrum is $P_{\mathrm{g}}(k)=b_{1}^{2} P(k)$ [see Heavens, Matarrese \& Verde (1998) for a discussion of this point]; $J$ is a function that depends on the shape of the triangle in $\boldsymbol{k}$-space, but only very weakly on cosmology (e.g. Bouchet et al. 1992, 1995; Catelan et al. 1995). Note that we assume a deterministic bias; other authors (e.g. Scherrer \& Weinberg 1998; Dekel \& Lahav 1999; Taruya et al. 1999) have investigated stochastic bias, where there is a random component to the relationship between $\delta$ and $\delta$. In the case of stochastic bias the bispectrum still has the form of equation (3), but the function $J$ is modified into $J^{\prime}$ in such a way that when the correlation coefficient of the stochastic bias $r$ goes to unity (i.e. the bias becomes deterministic), $J^{\prime} \rightarrow$ $J$. Theoretical considerations suggest that $r \sim 1$ on scales of interest (e.g. Tegmark \& Peebles 1998; Blanton et al. 2000; Seljak 2000).

In redshift space, both the power spectrum and the bispectrum are modified by redshift-space distortions, arising because the distance estimator (the redshift) is perturbed by peculiar velocities. These distortions are radial in nature, and can be analysed, at some expense in complexity, using radial and angular basis functions (e.g. Fisher, Scharf \& Lahav 1994; Heavens \& Taylor 1995; Ballinger, Heavens \& Taylor 1995; Hamilton 1998; Tadros et al. 1999). Here, we adopt the distant-observer approximation (Kaiser 1987), and assume that non-linear effects can be modelled by an incoherent small-scale velocity field, characterized by the pairwise velocity dispersion $\sigma_{\mathrm{p}}$. The large-scale infall leads to distortions that depend on the redshift distortion parameter $\beta=\Omega_{\mathrm{m}}^{0.6} / b_{1}$, where $\Omega_{\mathrm{m}}$ is the matter density parameter. Assuming in addition an exponential distribution for the pairwise velocity, the combined effect gives the power spectrum in redshift space (denoted by subscript $\mathrm{s}$ )

$P_{\mathrm{s}}(\boldsymbol{k})=\frac{P(k)\left(1+\beta \mu^{2}\right)^{2}}{1+k^{2} \mu^{2} \sigma_{\mathrm{p}}^{2} / 2}$

(e.g. Ballinger, Peacock \& Heavens 1996; Hatton \& Cole 1998), where $\mu$ is the cosine of the wavevector to the line of sight, which is a fixed direction in the distant-observer approximation. Other modifications have been suggested, such as a Gaussian, or exponential one-particle velocity dispersion, which yield different functional forms for the redshift distortion. Note that $\sigma_{\mathrm{p}}$ is usually written, as here, implicitly divided by the Hubble constant. The bispectrum is modified similarly, and again various modifications have been proposed. We use the form

$$
\begin{aligned}
B_{\mathrm{s}}\left(\boldsymbol{k}_{1}, \boldsymbol{k}_{2}, \boldsymbol{k}_{3}\right)= & \left(B_{12}+B_{23}+B_{31}\right)\left[\left(1+\frac{\alpha_{V}^{2} k_{1}^{2} \mu_{1}^{2} \sigma_{\mathrm{p}}^{2}}{2}\right)\right. \\
& \left.\times\left(1+\frac{\alpha_{V}^{2} k_{2}^{2} \mu_{2}^{2} \sigma_{\mathrm{p}}^{2}}{2}\right)\left(1+\frac{\alpha_{V}^{2} k_{3}^{2} \mu_{3}^{2} \sigma_{\mathrm{p}}^{2}}{2}\right)\right]^{-1 / 2}
\end{aligned}
$$

where

$B_{12} \equiv\left(1+\beta \mu_{1}^{2}\right)\left(1+\beta \mu_{2}^{2}\right)\left[\frac{\operatorname{Ker}\left(\boldsymbol{k}_{1}, \boldsymbol{k}_{2}\right)}{b_{1}}+\frac{b_{2}}{b_{1}^{2}}\right] P_{\mathrm{g}}\left(\boldsymbol{k}_{1}\right) P_{\mathrm{g}}\left(\boldsymbol{k}_{2}\right)$

and the kernel function Ker is $J$ modified for redshift space (see Verde et al. (1998), equation 13 for the formula); $\alpha_{V}$ is an adjustable parameter which is shape-dependent, and must be calibrated from simulations. Scoccimarro et al. (1999) propose an alternative ${ }^{1}$ for the denominator of (6), namely

$\left[1+\alpha_{S}^{2}\left(k_{1}^{2} \mu_{1}^{2}+k_{2}^{2} \mu_{2}^{2}+k_{3}^{2} \mu_{3}^{2}\right) \sigma_{v}^{2} / 2\right]^{2}$,

where the one-particle dispersion $\sigma_{v}=\sigma_{\mathrm{p}} / \sqrt{2}$ if the small-scale velocities are incoherent, and again the parameter $\alpha_{S}$ needs to be calibrated for different triangle shapes and cosmologies. We find that the formula (6) recovers the true bias parameter in an ensemble of simulated biased 2dFGRS catalogues with smaller scatter than (8). We see from (6) and (7) how the bispectrum can allow us to measure the bias parameters. The left-hand side is potentially observable, and we can hope to constrain $b_{1}$ and $b_{2}$ by considering triangles of different shape (and hence different Ker).

\section{METHOD}

The previous section shows the theoretical model for the bispectrum, and its dependence on the two parameters $b_{1}$ and $b_{2}$; note that, apart from these two parameters, the bispectrum depends on observable quantities such as $\beta, \sigma_{\mathrm{p}}$ and $P_{\mathrm{g}}$. The real-space galaxy power spectrum is obtained from the angle-averaged redshift-space power (cf. Verde et al. 1998):

$$
\begin{aligned}
P(k)= & P_{\mathrm{s}}(k)\left[4 \frac{\left(\sigma_{\mathrm{p}}^{2} k^{2}-\beta\right) \beta}{\sigma_{\mathrm{p}}^{4} k^{4}}+\frac{2 \beta^{2}}{3 \sigma_{\mathrm{p}}^{2} k^{2}}\right. \\
& \left.+\frac{\sqrt{2}\left(k^{2} \sigma_{\mathrm{p}}^{2}-2 \beta\right)^{2}}{k^{4} \sigma_{\mathrm{p}}^{5}} \tan ^{-1}\left(\frac{k \sigma_{\mathrm{p}}}{\sqrt{2}}\right)\right]^{-1} .
\end{aligned}
$$

The real-space power spectrum obtained in this way agrees well with the APM power estimated by Baugh \& Efstathiou (1994) and Efstathiou \& Moody (2001). The input catalogue is based on a revised and extended version of the APM galaxy catalogue (Maddox et al. 1990a).

The bispectrum and power spectrum data come from transforming the galaxy distribution as follows. The galaxies are weighted with the optimum weight for measuring the power spectrum (Feldman, Kaiser \& Peacock 1994), which also minimizes the variance of higher-order correlation functions (Scoccimarro 2000). The weight is $w(\boldsymbol{r})=1 /\left[1+P_{0} \bar{n}(\boldsymbol{r})\right]$, where $\bar{n}(\boldsymbol{r})$ is the average number density of galaxies at position $\boldsymbol{r}$ and $P_{0}$ is the power spectrum to be estimated. For reasons of computational speed, $P_{0}$ was fixed at $5000 \mathrm{~h}^{-3} \mathrm{Mpc}^{3}$ so that a fast Fourier transform could be employed. This is optimal for minimizing the variance at $k \simeq 0.1 h \mathrm{Mpc}^{-1}$, whereas our signal comes from wavenumbers with a smaller power, but in fact altering $P_{0}$ hardly changes the results.

\subsection{Estimating the bispectrum}

In this section we describe how we estimate the bispectrum from the data, taking into account the survey shape, selection function and shot noise.

${ }^{1}$ Note that in equation (38) of Scoccimarro et al. (1999) there is an extra power of 2 outside the round brackets, which we omit in (8). 
We follow Feldman et al. (1994) and Matarrese et al. (1997), and transform the field

$F(\boldsymbol{r}) \equiv \lambda w(\boldsymbol{r})\left[n(\boldsymbol{r})-\alpha n_{\mathrm{r}}(\boldsymbol{r})\right]$,

where $\lambda$ is a constant to be determined, $n_{\mathrm{r}}(\boldsymbol{r})$ is the number density of a random catalogue with the same selection function as the real catalogue, but with $1 / \alpha$ times as many particles. If we set $\lambda=I_{22}^{-1 / 2}$, where

$I_{i j} \equiv \int \mathrm{d}^{3} w^{i}(\boldsymbol{r}) \bar{n}^{j}(\boldsymbol{r})$

(Matarrese et al. 1997), then the power spectrum may be estimated from

$\left\langle\left|F_{k}\right|^{2}\right\rangle=P_{g}(\boldsymbol{k})+\frac{I_{21}}{I_{22}}(1+\alpha)$

and the bispectrum from

$$
\begin{aligned}
\left\langle F_{\boldsymbol{k}_{1}} F_{\boldsymbol{k}_{2}} F_{\boldsymbol{k}_{3}}\right\rangle= & \frac{I_{33}}{I_{22}^{3 / 2}}\left\{B_{\mathrm{g}}\left(\boldsymbol{k}_{1}, \boldsymbol{k}_{2}, \boldsymbol{k}_{3}\right)+\frac{I_{32}}{I_{33}}\left[P_{\mathrm{g}}\left(\boldsymbol{k}_{1}\right)+P_{\mathrm{g}}\left(\boldsymbol{k}_{2}\right)\right.\right. \\
& \left.\left.+P_{\mathrm{g}}\left(\boldsymbol{k}_{3}\right)\right]+\left(1-\alpha^{2}\right) \frac{I_{31}}{I_{33}}\right\} .
\end{aligned}
$$

An underlying assumption is that the power spectrum is roughly constant over the width of the survey window function in $\boldsymbol{k}$-space. Because of the rather flat geometry of the survey regions, and the holes owing to star drills, this criterion is not satisfied in detail. In practice, we have used multiple mock catalogues Cole et al. (1998) with the same selection criteria as the survey regions to check that this assumption does not feed through into a biased estimate of the bias parameters. Also, the power spectrum and bispectrum estimates are convolved with the window function. This can lead to changes in shape from convolution, and an erroneous interpretation of correlated noise as real features in the power spectrum. These effects are, however, not important in the wavenumber range $(0.1 \lesssim k \lesssim$ $0.5 h \mathrm{Mpc}^{-1}$ ) which we use for the bispectrum analysis (Percival et al. 2001).

To compute $F_{k}$, we generated random catalogues with approximately 5 times as many particles as the real catalogue, and analysed the North and South Galactic Pole regions (NGP and SGP) separately. We ignored the random fields present in the 2dFGRS as these complicate the window function and add very little information for the current analysis. Fast Fourier transforms were performed on a $512 \times 512 \times 256$ grid which encompassed all the particles, leading to a grid spacing of about $1 h^{-1} \mathrm{Mpc}$.

\subsection{Choice of triangles}

We use the real parts of $F_{\boldsymbol{k}_{1}} F_{\boldsymbol{k}_{2}} F_{\boldsymbol{k}_{3}}$ as our data, for triangles in $\boldsymbol{k}$ space (i.e. where $\boldsymbol{k}_{1}+\boldsymbol{k}_{2}+\boldsymbol{k}_{3}=\mathbf{0}$ ). Each triangle allows us to estimate a linear combination of the parameters natural to this analysis: $c_{1} \equiv 1 / b_{1}$ and $c_{2} \equiv b_{2} / b_{1}^{2}$, through equations (6) and (7). Note that we must use triangles of different shape (and hence different $\left.\operatorname{Ker}\left[\boldsymbol{k}_{1}, \boldsymbol{k}_{2}\right]\right)$ to lift the degeneracy between non-linear gravity and non-linear bias. As explained in the introduction, this is equivalent to analysing the shapes of structures that are different in the two cases.

Clearly, there are a huge number of possible triangles to investigate (many millions), and we are faced with a problem of how to choose the triangles to analyse. The problem is that triangles that have a wavevector in common will be correlated, through crossterms in the six-point function. A likelihood analysis as originally suggested by Matarrese et al. (1997) and Verde et al. (1998) with millions of correlated data points is infeasible, so we take a different approach. We use two sets of triangles of different configurations: one set with one wavevector twice the length of another, and another set with two wavevectors of common length. For the former, calibrations with mock catalogues give $\alpha_{V}=1.8$, and the latter $\alpha_{V}=1.0$. We set a lower limit to the wavenumber range of $k=0.1 \mathrm{~h} \mathrm{Mpc}^{-1}$, to avoid the effects of convolution with the window function (Percival et al. 2001). We set an upper limit of $k=0.5 \mathrm{~h} \mathrm{Mpc}^{-1}$ where, for the $2 \mathrm{dFGRS}$ power spectrum, the shot noise begins to dominate the signal so that there is little further to be gained by increasing the limit. In addition, we impose a constraint $k<0.35 h \mathrm{Mpc}^{-1}$ for the second configuration choice, where perturbation theory for this configuration begins to break down. This leaves us with $80 \times 10^{6}$ triangles. Two considerations motivate us to consider 'only' these $80 \times 10^{6}$ triangles: (i) adding more highly correlated triangles complicates the analysis significantly and does not add much signal and (ii) more importantly, only these two configurations have been extensively tested against fully non-linear $N$-body simulations. In fact, not only may perturbation theory breakdown on different scales depending on the triangle configuration, but also the (shape-dependent) redshift space distortion parameter $\alpha_{V}$ (equation 6) has only been calibrated for these two configurations.

To cope with so many triangles, we define a new estimator for $b_{1}$ and $b_{2}$ in the Appendix. Although the estimator is not at minimum variance, it is unbiased and has the big advantage in that it allows us to analyse many triangles, thus increasing the signal-to-noise ratio. The estimator does not give error bars; these are obtained by Monte Carlo simulation from 16 mock 2dFGRS catalogues (see Section 4). In Fig. 1 we show the measured (redshift-space) 2dFGRS bispectrum from the SGP and NGP for the two chosen triangle configurations. The dotted line shows the perturbation theory prediction for $b_{1}=1, b_{2}=0$, while the dashed line shows the shot noise contribution. For the bispectrum, the shot noise contribution becomes dominant around $k=0.5 \mathrm{~h} \mathrm{Mpc}^{-1}$.

\section{MOCK CATALOGUES AND TESTS}

We have used 16 mock catalogues from a Hubble Volume $N$-body simulation with $\Omega_{\mathrm{m}}=0.3, \Omega_{\Lambda}=0.7$ (' $\Lambda$ CDM' model), with the same selection function as the $2 \mathrm{dF}$ Galaxy Redshift Survey (Cole et al. 1998). ${ }^{2}$ This includes both the radial selection function and an angular mask that reflects the varying completeness of the survey of 2001 February. The catalogues contain mock galaxies for which the positions are determined according to the prescription described in Cole et al. (1998). This is a two-parameter exponential model based on the final density field, i.e. it does not conform to our assumption of equation (3). We will show that nevertheless the bispectrum method recovers the bias parameter $b_{1}$, defined by the square root of the ratio of the galaxy and the matter power spectra. ${ }^{3}$ This is the crucial test, since it is this ratio that we wish to determine, as we can then translate the galaxy power spectrum to the underlying mass spectrum. For the simulated galaxies in the Hubble volume as a whole the square root of the power spectrum ratio varies between about 0.9 at large scales and 0.75 at $k=0.5 h \mathrm{Mpc}^{-1}$. Over the scales probed by our

\footnotetext{
${ }^{2}$ See http://star-www.dur.ac.uk/ cole/mocks/hubble.html We find that 16 mock catalogues are sufficient to estimate the error bars. The choice of the cosmological model is not important for the error estimate, however, this choice of the cosmological model turns out to be not too far away from the model recovered a posteriori.

${ }^{3}$ For a more general discussion of definitions of bias, see Lahav et al. (2002).
} 


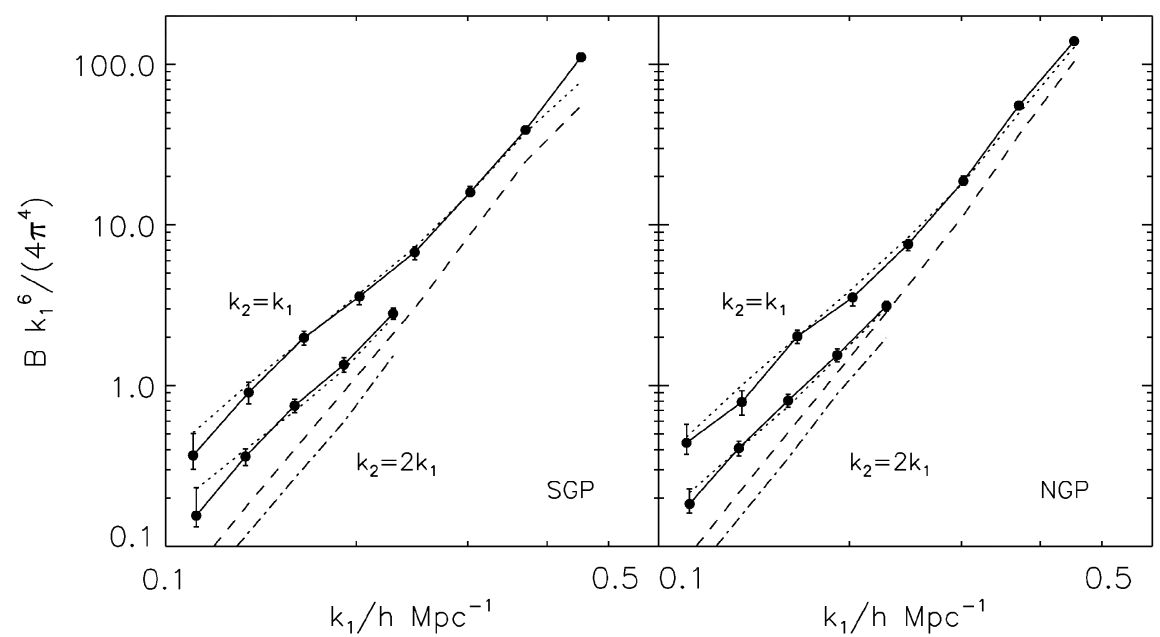

Figure 1. Measured (redshift-space) 2dFGRS dimensionless bispectrum from the SGP and NGP for the two chosen configurations. The dotted line shows the perturbation theory prediction for $b_{1}=1, b_{2}=0$ while the dashed and dot-dashed lines show the shot noise contributions. The error bars are obtained via Monte Carlo simulation of 16 mock 2dFGRS catalogues (see the text for details).
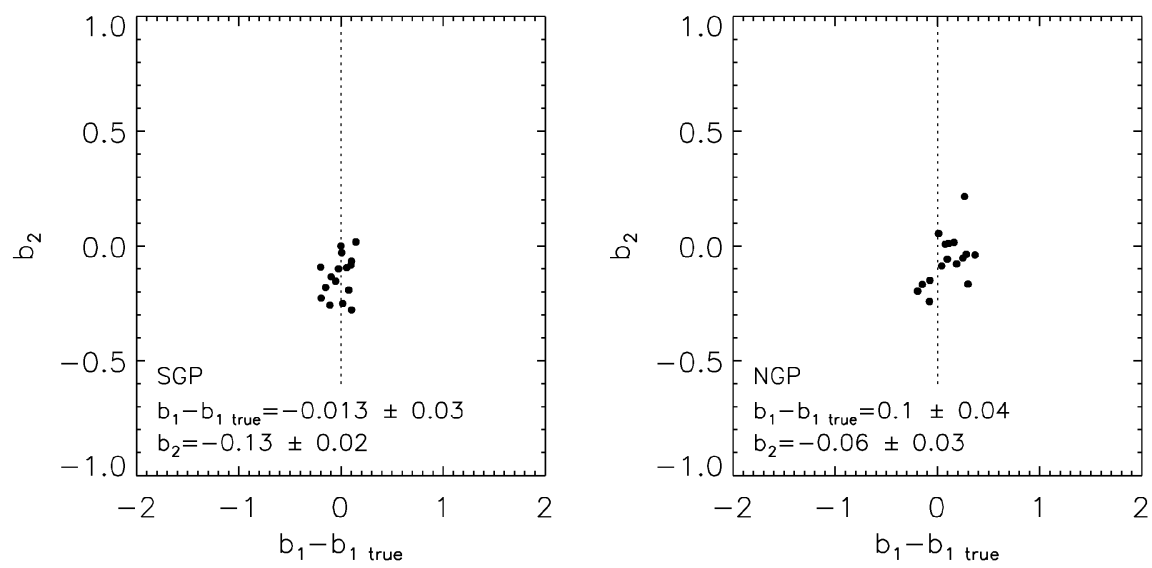

Figure 2. Error on the linear bias parameter from $\Lambda$ CDM mock galaxy catalogues in the SGP (left) and NGP (right). The average bias in the estimator is consistent with zero for the SGP: $-0.01 \pm 0.03$, but shows a small bias of $0.10 \pm 0.04$ for the NGP. The sample rms of 0.11 for the SGP and 0.16 for the NGP are used in the analysis of the 2dFGRS.

bispectrum triangles, the bias is roughly 0.8 , but with an uncertainty that ultimately limits our error determination. The power spectrum of the simulated 2dFGRS catalogues varies a little, so we estimate $b_{1} \equiv\left(P_{\mathrm{g}} / P\right)^{1 / 2}$ individually for each catalogue (and individually for SGP and NGP), from the wavenumber range $0.1-0.5 h \mathrm{Mpc}^{-1}$. This sets $\beta$ for each sample, and we fit the pairwise velocity dispersion $\sigma_{\mathrm{p}}$ individually by requiring a good fit to the redshift-space power spectrum, using the real-to-redshift mapping of equation (9).

We then analyse the set of triangles for each simulation, as detailed above, and in Fig. 2 we show the error in the bias from 16 simulations in the SGP and NGP. The average bias in the estimator is consistent with zero for the SGP: $-0.01 \pm 0.03$, but shows a small bias of $0.10 \pm 0.04$ for the NGP. ${ }^{4}$ Fig. 2 shows that the approximations made in the analysis (e.g. the functional form of the bias, the window function, the distant observer approximation, etc.) do not significantly bias the result. We will use the sample rms of

4 This is understandable since the NGP is smaller than the SGP, thus the effects of the convolution with the window are more important.
0.11 for the SGP and 0.16 for the NGP as the errors in the analysis of the $2 \mathrm{dFGRS}$. We also carried out the same analysis on 16 mock catalogues of the SGP obtained from the Hubble volume simulation for a $\tau$ CDM model. The true underlying bias parameter is correctly recovered with a 20 per cent error $(b=1.7 \pm 0.3)$. The error in the natural parameter $1 / b_{1}$ is comparable for the $\Lambda \mathrm{CDM}$ and $\tau \mathrm{CDM}$ models, but the value of $b_{1}$ itself is larger in the latter case. In Fig. 3 we show $b_{1}$ recovered with the bispectrum method versus the underlying (true) $b_{1} \equiv \sqrt{P_{\mathrm{g}} / P}$ for 16 mock SGP simulations for the $\tau \mathrm{CDM}$ and $\Lambda \mathrm{CDM}$ models.

\section{RESULTS}

We analyse the catalogue as compiled in 2001 February (i.e. the same catalogue as in Percival et al. 2001), and we analyse the NGP and SGP regions separately; we excise the random fields entirely. This leaves us with 75792 and 51862 galaxies in the SGP and NGP, respectively. The two survey regions give us a useful consistency check. 


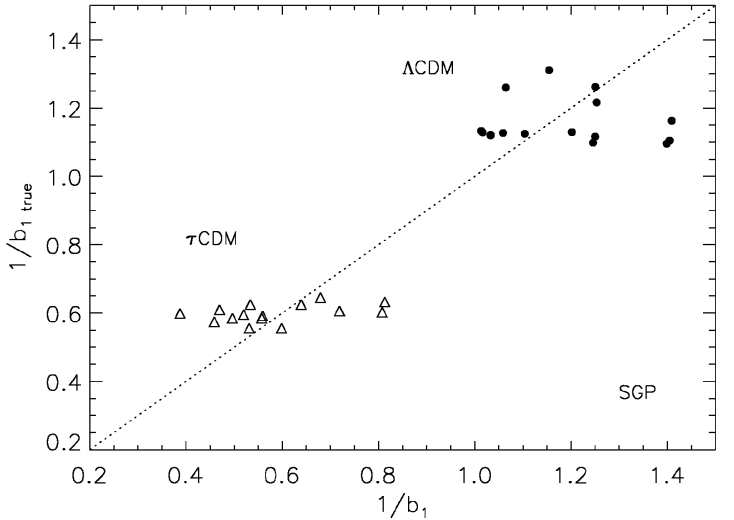

Figure 3. $1 / b_{1}$ recovered with the bispectrum method versus the underlying (true) $1 / b_{1} \equiv \sqrt{P / P_{\mathrm{g}}}$ for 16 mock SGP simulations for the $\tau \mathrm{CDM}$ and $\Lambda \mathrm{CDM}$ models. $1 / b_{1}$ is the natural quantity in the analysis of the bispectrum (see equation 4). Note that the 2dFGRS has data in the NGP and SGP, reducing the error bar compared with these mock catalogues.

We initially present results fixing $\beta=0.43$ and $\sigma_{\mathrm{p}}=385 /$ $H_{0} \mathrm{~km} \mathrm{~s}^{-1}\left(3.85 \mathrm{~h}^{-1} \mathrm{Mpc}\right)$. These are the best-fitting values from the analysis of the 2dFGRS redshift-space correlation function (Peacock et al. 2001; Norberg et al. 2001a; see also Tegmark, Hemilton \& Xu 2001). Note that this value of $\beta$ involves a bias parameter that is defined somewhat differently from ours, in terms of the correlation function. This bias parameter will coincide with our $b_{1}$ only if certain assumptions hold, such as if the overdensity in galaxies is everywhere $b_{1}$ times the mass overdensity. In due course, $\beta$ can be determined by analysis of the 2dFGRS power spectrum itself, but for the time being we assume that the two bias parameters are the same. Finally, we will marginalize over $\beta$ and $\sigma_{\mathrm{p}}$.

\subsection{Bias parameters}

It is difficult to find a method to display in a single figure all of the information from the $80 \times 10^{6}$ triangles used: for this reason we display only one configuration and for the SGP alone - adding other configurations (or the NGP) would complicate the figure. Fig. 4 shows the SGP data for configurations with two wavevectors of common length, along with the perturbation theory predictions for different combinations of the linear and quadratic bias parameters. The dashed and dot-dashed lines are the perturbation theory prediction for $b_{1}=1.3, b_{2}=0$ and $b_{1}=1.0, b_{2}=0.5$. The error bars come from the mock catalogues, and the offset between the centres of the error bars and the points shows the bias in the estimator.

Fixing the values of $\beta$ and $\sigma_{\mathrm{p}}$, and including all of the triangles in the SGP gives as a raw result $b_{1}=1.06, b_{2}=0.01$. The NGP linear bias is slightly higher: $b_{1}=1.12, b_{2}=-0.07$. Adjusting these results with the estimator bias obtained from the mock catalogues (Section 4), and associating an error from the mock catalogues gives

$b_{1}=1.07 \pm 0.11 \quad(\mathrm{SGP})$

$b_{1}=1.02 \pm 0.16 \quad(\mathrm{NGP})$

and a combined minimum-variance weighted result of

$b_{1}=1.05 \pm 0.09$.

Note that the estimator bias does not significantly alter the results: ignoring it increases the estimate by 0.03 , much less than the statistical error.

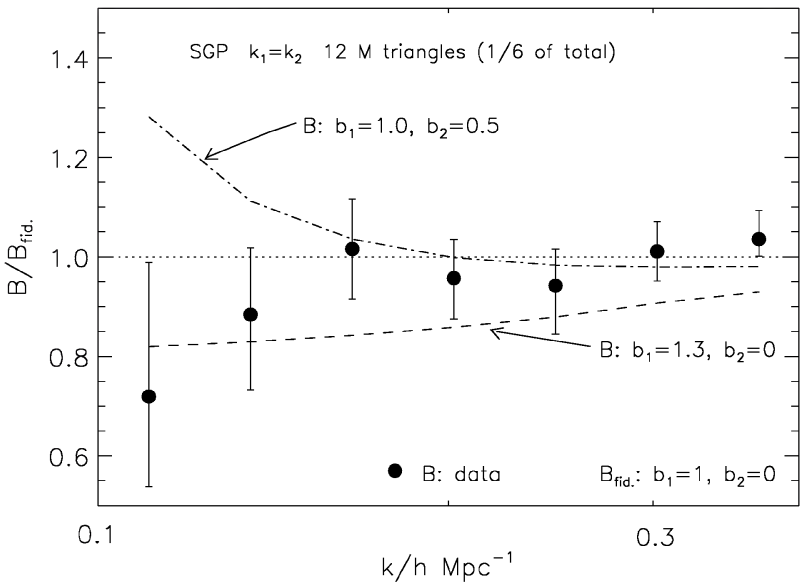

Figure 4. Ratio of the average measured bispectrum and the average perturbation theory predictions, relative to the bispectrum for a fiducial unbiased model $\left(B_{\mathrm{fid}}\right)$. Dashed line $b_{1}=1.3, b_{2}=0$, dot-dashed line $b_{1}=1.0$, $b_{2}=0.5$. To produce this figures only the SGP data were used and only configurations with two wavevectors of common length. This means that only 12 million triangles were used from the total 80 millions. Inclusion of the remainder excludes both models at high confidence. The error bars are obtained via Monte Carlo from the $16 \mathrm{~N}$-body simulations, and are placed centrally on the mean of the estimates from the mock catalogues. This illustrates the level of bias in the estimator. The figure also shows that there is no evidence of scale-dependent bias.

Our estimate of the quadratic bias parameter from the NGP and SGP 2dFGRS is

$b_{2}=-0.02 \pm 0.07$,

with SGP and NGP individually giving $b_{2}=0.01 \pm 0.09$ and $-0.07 \pm 0.11$, respectively.

Thus we see that the 2dFGRS galaxies are perfectly consistent with tracing the underlying mass distribution on these scales (wavelengths $\lambda=2 \pi / k=13-63 h^{-1} \mathrm{Mpc}$ ), i.e. $b_{1}$ is consistent with unity and $b_{2}$ with zero.

The results depend mildly on the values of $\beta$ and $\sigma_{\mathrm{p}}$ used. Marginalizing over the distribution of these quantities estimated from the redshift-space correlation function (Peacock et al. 2001), the final results are virtually unchanged: $b_{1}=1.040 .06, b_{2}=$ $-0.054 \pm 0.04$; adding these errors in quadrature with those of (15) gives our final estimates:

$b_{1}=1.04 \pm 0.11$

$b_{2}=-0.054 \pm 0.08$.

\subsection{Luminosity-dependent and scale-dependent bias}

The 2dFGRS exhibits luminosity-dependent clustering (Norberg et al. 2001a,b), so it is reasonable to ask in what sense the galaxies in the 2dFGRS are unbiased. We have consistently used all the galaxies in the $2 \mathrm{dFGRS}$ NGP and SGP regions to determine $\beta$ and the bias parameters. With the weighting scheme employed to measure $\beta$ and $b_{1}$, the weighted mean luminosity is $1.9 L_{*}$. From studies of the correlation function, Norberg et al. (2001a) found a luminosity dependence in the relative bias of 2dFGRS galaxies: $b / b_{*}=0.85+0.15 L / L_{*}$. If we use this relation to adjust our recovered bias to apply to $L_{*}$ galaxies, we find they could be slightly antibiased, but they are still consistent with $b_{1}=1\left(b_{1 *}=0.92 \pm 0.11\right.$ assuming $\left.b_{1}=b\right)$. 
The 2dFGRS galaxies, as a population, trace the mass extremely well.

Regarding the scale dependence of the bias, it is worth noting that theoretical arguments (e.g. Mann, Peacock \& Heavens 1998; Peacock \& Smith 2000; Seljak 2000; Benson et al. 2000; Blanton et al. 2000; Berlind \& Weinberg 2001) suggest that bias is expected to be constant on large scales (larger than a few $h^{-1} \mathrm{Mpc}$ ) and a scaledependent bias is plausible on intermediate scales, but the scale dependence is expected to be weak. We find no evidence of scaledependent bias (see Fig. 4). Both scale and luminosity dependence may be explored in detail when the catalogue is complete.

\subsection{The matter density of the Universe}

Analysis of the redshift-space correlation function allows one to estimate $\beta=\Omega_{\mathrm{m}}^{0.6} / b_{1}$ (Peacock et al. 2001), and this can be combined with our determination of $b_{1}$ to estimate the matter density parameter $\Omega_{\mathrm{m}}$. A strength of the result is that it is obtained from the $2 \mathrm{dFGRS}$ alone, independent of all other data sets. Following the same procedure as in the previous subsection, we marginalize over the uncertainty in $\beta$ and $\sigma_{\mathrm{p}}$, to obtain

$\Omega_{\mathrm{m}}=0.27 \pm 0.06$.

The small size of the error bar is worth remarking on. For each pair of values of $\beta$ and $\sigma_{\mathrm{p}}$, we obtain an estimate of $b_{1}$. The estimates of $\beta$ and $b_{1}$ are slightly anticorrelated, which leads to a slightly smaller scatter in $\Omega_{m}$ than one would expect from the errors on $\beta$ and $b_{1}$.

This result is consistent with other recent determinations by other methods such as combining CMB with large-scale structure or supernova measurements (e.g. Percival et al. 2001; Jaffe et al. 2001; Efstathiou et al. 2002) and from early weak lensing measurements (Hoekstra, Yee \& Gladders 2001).

\subsection{Comparison with previous work}

Previous work has concentrated on using cumulants, although there have been two analyses (Feldman et al. 2001; Scoccimarro et al. 2001) of the bispectrum of infrared-selected galaxies observed with the Infrared Astronomy Satellite (IRAS; Fisher et al. 1995; Saunders et al. 2000). The IRAS samples are much smaller than the current 2dFGRS, and are also rather shallow, so shot noise and the radial nature of the redshift distortion is more severe than for 2dFGRS. Nevertheless, bias estimates have been made, and reported with remarkably small error bars. The most accurate quoted values are from Feldman et al. (2001), $1 / b_{1}=1.2_{-0.19}^{+0.18}$ for the PSCz survey (Saunders et al. 2000). This value of the linear bias parameter is consistent with our determinations, since IRAS galaxies have a power spectrum that is lower than optically selected galaxies, by a factor $\sim 1.3^{2}$ on the relevant scales (Peacock \& Dodds 1994; Seaborne et al. 1999).

Studies of the skewness, kurtosis and higher-order moments of the optical galaxy distribution (e.g. Gaztanaga 1994; Szapudi \& Szalay 1997; Hui \& Gaztanaga 1999; Hoyle, Szapudi \& Baugh 2000) have also shown consistency with a linear bias of unity, but here we are able to derive both the linear bias and the quadratic bias simultaneously. In addition, our present results are more accurate and convert these general indications of a low degree of bias into a strong constraint on theoretical models.

\section{SUMMARY AND CONCLUSIONS}

We have demonstrated through analysis of the bispectrum of the 2dFGRS that the optically selected galaxies of the sample trace the matter density extremely well on large scales (Fourier modes with $0.1<k<0.5 h \mathrm{Mpc}^{-1}$ that correspond approximately to $30<r<5 h^{-1} \mathrm{Mpc}$ ). Specifically, the linear bias parameter is very close to unity, and the quadratic (non-linear) bias is very close to zero. Ironically, this is exactly the assumption that used to be made decades ago in large-scale structure analysis, but which was questioned when the concept of biased galaxy formation was introduced (e.g. Kaiser 1984; Peacock \& Heavens 1985; Bardeen et al. 1986; Dekel \& Rees 1994). Theoretical arguments suggest that an initial bias at formation approaches unity with time, provided that galaxies are neither created nor destroyed (Fry 1996), and that the Universe does not become curvature- or vacuum-dominated in the meantime (Catelan, Matarrese \& Porciani 1998). In any case, these assumptions will clearly fail at some level. Note that the effective depth of the survey is $z=0.17$ and our measurement should be interpreted as the bias and $\Omega_{\mathrm{m}}$ at this epoch (for an additional discussion see Lahav et al. 2002). Because of a tendency for the bias to approach unity with time, with this measurement we do not rule out a significant bias of galaxies at formation time, but the unbiased nature of the galaxies today puts a significant constraint on theoretical models of galaxy formation.

Currently, we find no evidence of scale-dependent bias (see Fig. 4). The size of the survey at the present time does not allow us to place strong constraints on the scale dependence or the luminosity dependence of the bias parameter, or on the nature of biasing (e.g. deterministic, stochastic, Eulerian, Lagrangian, etc.), but these issues will be explored with the completion of the survey. At some level non-linear bias must appear on small scales, from the morphology-density relation (Dressler 1980; Hashimoto \& Oemler 1999), but this is probably on too small scales for the perturbative method of this paper to be valid.

Our measurement of the matter density of the Universe $\Omega_{\mathrm{m}}=0.27 \pm 0.06$ should be interpreted as $\Omega_{\mathrm{m}}$ at the effective redshift of the survey. The extrapolation at $z=0$ is model-dependent, but the changes this correction introduces are below the quoted error bars for reasonable choices of model. Our measurement is in agreement with other independent determinations such as cosmic microwave background together with Hubble constant constraints (e.g. Jaffe et al. 2001; Efstathiou et al. 2002; Freedman et al. 2001) that also give comparable error bars.

It is worth re-emphasizing that the analysis presented here relies only on the 2dFGRS data set. It is also important that we not only conclude that the linear bias is essentially unity, but also that the quadratic (non-linear) bias term is constrained to be very close to zero. Taken together, these measurements argue powerfully that the 2dFGRS galaxies do indeed trace the mass on large scales. In addition to our findings, joint analysis of the 2dFGRS and CMB data (Lahav et al. 2002) also supports our conclusion that the 2dFGRS galaxies are unbiased. Different methods of measuring linear bias require different assumptions, and it is remarkable that such different methods agree on the basic cosmological model.

\section{ACKNOWLEDGMENTS}

LV is supported in part by NASA grant NAG5-7154, and acknowledges the University of Edinburgh for hospitality. AFH thanks Rutgers and Princeton Universities for hospitality. We are grateful to Raul Jimenez and David Spergel for useful discussions. The $2 \mathrm{dF}$ Galaxy Redshift Survey was made possible through the dedicated efforts of the staff of the Anglo-Australian Observatory, both in creating the $2 \mathrm{dF}$ instrument and in supporting it on the telescope. 


\section{REFERENCES}

Ballinger W. E., Heavens A. F., Taylor A.N., 1995, MNRAS, 276, 59P Ballinger W. E., Peacock J. A., Heavens A. F., 1996, MNRAS, 282, 877

Bardeen J. M., Bond J. R., Kaiser N., Szalay A. S., 1986, ApJ, 304, 15

Baugh C. M., Efstathiou G., 1994, MNRAS, 267, 323

Benson A. J., Cole S., Frenk C. S., Baugh C. M., Lacey C. G., 2000 , MNRAS, 311, 793

Berlind A. A., Weinberg D. H., 2001, astro-ph/0109001

Blanton M., Cen R., Ostriker J. P., Strauss M. A., Tegmark M., 2000, ApJ, 531,1

Bouchet F., Juszkiewicz R., Colombi S., Pellat R., 1992, ApJ, 394, L5

Bouchet F., Colombi S., Hivon E., Juszkiewicz R., 1995, A\&A, 296, 575

Catelan P., Lucchin F., Matarrese S., Moscardini L., 1995, MNRAS, 276, 39

Catelan P., Moscardini L., 1994, ApJ, 426, 14

Catelan P., Matarrese S., Porciani C., 1998, ApJ, 502, L1

Cole S., Hatton S., Weinberg D. H., Frenk C. S., 1998, MNRAS, 300, 945

Colless M. et al., 2001, MNRAS, 328, 1039

Davis M., Efstathiou G., Frenk C. S., White S. D. M., 1985, ApJ, 292, 371

Dekel A., Lahav O., 1999, ApJ, 520, 24

Dekel A., Rees M. J., 1994, ApJ, 422, L1

Dressler A., 1980, ApJ, 236, 351

Efstathiou G., Moody S. J., 2001, MNRAS, 325, 1603

Efstathiou G. P. et al., 2002, MNRAS, 330, 29

Feldman H. A., Kaiser N., Peacock J. A., 1994, ApJ, 426, 23

Feldman H. A., Frieman J. A., Fry N. J., Scoccimarro R., 2001, Phys. Rev. Lett., 86, 1434

Fisher K. B., Huchra J. P., Strauss M. A., Davis M., Yahil A., Schlegel D., 1995, ApJ Suppl., 100, 69

Fisher K. B., Scharf C., Lahav O., 1994, MNRAS, 266, 219

Freedman W. L. et al., 2001, ApJ, 553, 47

Fry J. N., 1994, Phys. Rev. Lett., 73, 215

Fry J. N., 1996, ApJ, 461, L65

Fry J. N., Gaztanaga E., 1993, ApJ, 413, 447

Gaztanaga E., 1994, MNRAS, 268, 913

Hamilton A. J. S., 1988, ApJ, 331, L59

Hamilton A. J. S., 1998, in Hamilton D., ed. Ringberg Workshop on LargeScale Structure. Kluwer Academic, Dordrecht,, 185

Hashimoto Y., Oemler A. J., 1999, ApJ, 510, 609

Hatton S. J., Cole S., 1998, MNRAS, 296, 10

Heavens A. F., Taylor A. N., 1995, MNRAS, 275, 483

Heavens A. F., Matarrese S., Verde L., 1998, MNRAS, 301, 797

Hivon E., Bouchet F., Colombi S., Juszkiewicz R., 1995, A\&A, 298, 643

Hoekstra H., Yee H., Gladders M., 2001, ApJ, 558, 11

Hoyle F., Szapudi I., Baugh C. M., 2000, MNRAS, 317, L51

Hui L., Gaztanaga E., 1999, ApJ, 519, 622

Jaffe A. et al., 2001, Phys. Rev. Lett., 86, 3475

Kaiser N., 1984, ApJ, 284, L9

Kaiser N., 1987, MNRAS, 227, 1

Komatsu E., Wandelt B., Spergel D., Banday A., Gorski K., 2002, ApJ, 566, 19

Lahav O., Saslaw W. C., 1992, ApJ, 396, 430

Lahav O., Nemiroff R. J., Piran T., 1990, ApJ, 350, 119

Lahav O. et al., 2002, MNRAS, 333, 961

Lewis I. et al., 2002, MNRAS, in press (astro-ph/0203336)

Maddox S. J., Sutherland W. J., Efstathiou G., Loveday J., 1990, MNRAS, 243, 692

Mann R. G., Peacock J. A., Heavens A. F., 1998, MNRAS, 293, 209

Matarrese S., Verde L., Heavens A., 1997, MNRAS, 290, 651

Norberg P. et al., 2001a, MNRAS, 328, 64

Norberg P. et al., 2001b, MNRAS, 332, 827

Peacock J. A., Dodds S. J., 1994, MNRAS, 267, 1020

Peacock J. A., Heavens A. F., 1985, MNRAS, 217, 805

Peacock J. A., Smith R. E., 2000, MNRAS, 318, 1144

Peacock J. A. et al., 2001, Nat, 410, 169

Percival W. et al., 2001, MNRAS, 327, 1297

Postman M., Geller M. J., 1984, ApJ, 281, 95

Santos M. et al., 2001, astro-ph/0107588
Saunders W. et al., 2000, MNRAS, 317, 55

Scherrer R. J., Weinberg D. H., 1998, ApJ, 504, 607

Scoccimarro R., 2000, ApJ, 544, 597

Scoccimarro R., Colombi S., Fry J. N., Frieman J. A., Hivon E., Melott A., 1998, ApJ, 496, 586

Scoccimarro R., Couchman H., Frieman J. A., 1999, ApJ, 517, 531

Scoccimarro R., Feldman H. A., Fry J. N., Frieman J. A., 2001, ApJ, 546, 652

Seaborne M. D. et al., 1999, MNRAS, 309, 89

Seljak U., 2000, MNRAS, 318, 203

Smoot G. F. et al., 1992, ApJ, 396, L1

Szapudi I., Szalay A. S., 1997, ApJ, 481, L1

Tadros H. et al., 1999, MNRAS, 305, 527

Taruya A., Koyama K., Soda J., 1999, ApJ, 510, 541

Tegmark M., Peebles P. J. E., 1998, ApJ, 500, 79

Tegmark M., Hamilton A. J. S., Xu Y., 2001, astroph, 0111575

Verde L., Heavens A., Matarrese S., Moscardini L., 1998, MNRAS, 300, 747

Verde L., Wang L., Heavens A., Kamionkowski M., 2000, MNRAS, 313, 141

White S. D. M., Tully R. B., Davis M., 1988, ApJ, 333, L45

Zeldovich Y. B., 1970, A\&A, 5, 84

\section{APPENDIX}

Here we outline the new estimator for the bias parameters. Although not optimal, it allows many millions of triangles to be analysed.

The likelihood of $c_{1} \equiv 1 / b_{1}$ and $c_{2} \equiv b_{2} / b_{1}^{2}$ from each triangle (labelled by $\gamma$ ) is degenerate, since it constrains only a linear combination of $c_{1}$ and $c_{2}$, namely

$B_{\gamma}=R_{\gamma} c_{1}+S_{\gamma} c_{2}+T_{\gamma}$,

where the expressions for $R_{\gamma}, S_{\gamma}$ and $T_{\gamma}$ are at the end of this Appendix. The bispectrum estimate $B_{\gamma}$ has an intrinsic statistical error $\sigma_{\gamma}$ as discussed (e.g. in Matarrese et al. 1997). In computing the bias parameter, we wish to weight the determination obtained from each triangle by the inverse of the variance of the corresponding bispectrum. Since this is a cumbersome expression (see Matarrese et al. 1997; Verde et al. 1998) we approximate this by its dominant Gaussian term,

$\left\langle\left|F_{k_{1}} F_{k_{2}} F_{k_{3}}\right|^{2}\right\rangle=\left(P_{\mathrm{g} 1}+P_{\mathrm{SN}}\right)\left(P_{\mathrm{g} 2}+P_{\mathrm{SN}}\right)\left(P_{\mathrm{g} 3}+P_{\mathrm{SN}}\right)$,

where $P_{\mathrm{g} 1}=P_{\mathrm{g}}\left(k_{1}\right)$, etc. and $P_{\mathrm{SN}}$ is the shot noise. The inclusion of higher-order terms leaves the results practically unchanged for this data set, and adds considerably to the computing time. If we now assume that this error is Gaussian distributed we can immediately see that the likelihood contours will be lines in the $c_{1}-c_{2}$ plane:

$\ln \mathcal{L}\left(c_{1}, c_{2}\right) \propto-\ln \sigma_{\gamma}-\frac{\left(B_{\gamma}-R_{\gamma} c_{1}+S_{\gamma} c_{2}+T_{\gamma}\right)^{2}}{2 \sigma_{\gamma}^{2}}$.

This should give an unbiased a posteriori probability for $c_{1}$ and $c_{2}$, if we assume uniform priors. We define a non-optimal estimator by simply multiplying these likelihoods together, ignoring the correlations between different triangles, forming what we term the pseudolikelihood. The maximum of the pseudolikelihood is still asymptotically unbiased. The error, however, cannot be determined internally; we estimate it by Monte Carlo methods, i.e. from the dispersion of $c_{1}$ and $c_{2}$ estimates from 16 mock catalogues. This procedure also allows us to see whether the estimator is biased or not. Moreover, by estimating the errors via the Monte Carlo method, our final estimates do not depend on the initial assumption of Gaussian likelihood.

The assumption of Gaussian likelihood, however, makes the maximum of the pseudolikelihood calculable analytically, yielding the following estimates for $c_{1}$ and $c_{2}$ : 
$\hat{c}_{1}=\frac{2 A_{1} A_{22}-A_{2} A_{12}}{A_{12}^{2}-4 A_{11} A_{22}}$

$\hat{c}_{2}=\frac{2 A_{2} A_{11}-A_{1} A_{12}}{A_{12}^{2}-4 A_{11} A_{22}}$,

where

$A_{11} \equiv \sum_{\gamma} \frac{R_{\gamma}^{2}}{2 \sigma_{\gamma}^{2}}$

$A_{22} \equiv \sum_{\gamma} \frac{S_{\gamma}^{2}}{2 \sigma_{\gamma}^{2}}$

$A_{1} \equiv \sum_{\gamma} \frac{\left(T_{\gamma}-B_{\gamma}\right) R_{\gamma}}{\sigma_{\gamma}^{2}}$

$A_{2} \equiv \sum_{\gamma} \frac{\left(T_{\gamma}-B_{\gamma}\right) S_{\gamma}}{\sigma_{\gamma}^{2}}$

$A_{12} \equiv \sum_{\gamma} \frac{R_{\gamma} S_{\gamma}}{\sigma_{\gamma}^{2}}$

We can also, if desired, compute $c_{1}$ on the assumption that $c_{2}=0$ :

$\hat{c}_{1}=-\frac{A_{1}}{2 A_{11}} \quad\left(c_{2} \equiv 0\right)$.

(A11)

The expressions for $R_{\gamma}, S_{\gamma}$ and $T_{\gamma}$ can easily be obtained from the expression for the bispectrum of Verde et al. (1998) (Sections 2.3 and 2.4). We report them here for completeness. To obtain their corresponding real-space quantities just set $\sigma_{v}=0$ and $\beta=0$ :

$$
\begin{aligned}
R_{\gamma}= & \left\{\left[J\left(\boldsymbol{k}_{1}, \boldsymbol{k}_{2}\right)+\mu^{2} \beta K\left(\boldsymbol{k}_{1}, \boldsymbol{k}_{2}\right)\right] 2 P_{\mathrm{g}}\left(k_{1}\right) P_{\mathrm{g}}\left(k_{2}\right)\right. \\
& \left.\times\left(1+\beta \mu_{1}^{2}\right)\left(1+\beta \mu_{2}^{2}\right)+\text { cyc. }\right\} D_{3} .
\end{aligned}
$$

where $\mu=-\mu_{3}$ for the term explicitly written and the expression for the kernel $K$ can be found (e.g. in Catelan \& Moscerdini 1994),

$S_{\gamma}=\left[\left(1+\beta \mu_{1}^{2}\right)\left(1+\beta \mu_{2}^{2}\right) P_{\mathrm{g}}\left(k_{1}\right) P_{\mathrm{g}}\left(k_{2}\right)+\right.$ cyc. $] D_{3}$

$$
\begin{aligned}
T_{\gamma}= & \left\{\left[\mu_{1}^{2} \mu_{2}^{2} \beta+\frac{\beta}{2}\left(m u_{1}^{2}+\mu_{2}^{2}\right)+\beta \mu_{1} \mu_{2}\left(\frac{k_{1}}{k_{2}}+\frac{k_{2}}{k_{1}}\right)\right.\right. \\
& \left.+\frac{\beta^{2}}{2} \mu_{1} \mu_{2}\left(\mu_{1}^{2} \frac{k_{1}}{k_{2}}+\mu_{2}^{2} \frac{k_{2}}{k_{1}}\right)\right] 2 P_{\mathrm{g}}\left(k_{1}\right) P_{\mathrm{g}}\left(k_{2}\right) \\
& \left.\times\left(1+\beta \mu_{1}^{2}\right)\left(1+\beta \mu_{2}^{2}\right)+\text { cyc. }\right\} D_{3} \\
& +\left[P_{\mathrm{g}}\left(k_{1}\right) \frac{\left(1+\beta \mu_{1}^{2}\right)^{2}}{\left(1+\sigma_{v}^{2} / 2 k_{1}^{2} \mu_{1}^{2}\right)}+\text { cyc. }\right] \frac{I_{32}}{I_{33}}+\left(1-\alpha^{2}\right) \frac{I_{31}}{I_{33}},
\end{aligned}
$$

where $D_{3}$ denotes the damping term arising from the incoherent small-scale velocity dispersion (see equation 6):

$$
\begin{aligned}
D_{3}= & {\left[\left(1+\frac{\alpha_{V}^{2} k_{1}^{2} \sigma_{\mathrm{p}}^{2} \mu_{1}^{2}}{2}\right)\left(1+\frac{\alpha_{V}^{2} k_{2}^{2} \sigma_{\mathrm{p}}^{2} \mu_{2}^{2}}{2}\right)\right.} \\
& \left.\times\left(1+\frac{\alpha_{V}^{2} k_{3}^{2} \sigma_{\mathrm{p}}^{2} \mu_{3}^{2}}{2}\right)\right]^{-1 / 2} .
\end{aligned}
$$

This paper has been typeset from a $\mathrm{TEX}_{\mathrm{X}} / \mathrm{LT} \mathrm{T} \mathrm{X}$ file prepared by the author. 